\title{
Lattice Boltzmann simulation of power-law fluid flow in the mixing section of a single-screw extruder
}

\author{
J.M. Buick \\ Mechanical and Design Engineering, Anglesea Building, Anglesea Road University of Portsmouth, Portsmouth PO1 3DJ, UK
}

\section{A R T I C L E I N F O}

\section{Article history:}

Received 19 April 2008

Received in revised form 16 September 2008

Accepted 21 September 2008

Available online 5 October 2008

\section{Keywords:}

Numerical simulation

Non-Newtonian

Lattice Boltzmann model

Flow field

Single-screw extruder

\begin{abstract}
A B S T R A C T
The single-screw extruder is commonly used in polymer processing where the performance of the mixing section is significant in determining the quality of the final product. It is therefore of great interest to simulate the flow field in a single-screw extruder. In this paper simulations of non-Newtonian fluids in a single-screw extruder are performed using the lattice Boltzmann model.
\end{abstract}

(ㄷ) 2007 Elsevier Ltd. All rights reserved.

\section{Introduction}

A single-screw extruder is commonly used in polymer processing. The mixing performance of the extruder considerably influences the quality and morphology of the final product. For this reason the flow field in the mixing section has been studied by a number of authors to gain a better understanding of the process. Yao et al. $(1996,1997)$ used the finite difference method (FDM) to determine the flow field in a single-screw extruder geometry. The simulations were shown to be in good agreement with the results of a flow visualisation experiment using high viscosity corn syrup. Horiguchi et al. (2003) used the lattice gas method (LGM) to examine the same problem. The LGM results were found to be in good agreement with visualisation experiments. Horiguchi et al. (2003) also considered a quantitative comparison with theory. This indicated that the LGM produced a more accurate representation of the flow field compared to the FDM; however, there was still a discrepancy between the LGM simulation and the analytic expression. Simulations using the lattice Boltzmann model (LBM) were performed by Buick and Cosgorve (2006). The LBM is a simplified kinetic model (Chen and Doolen, 1998) which has developed from the LGM. The LBM was shown to simulate the flow in the single-screw extruder more accurately and more efficiently than the LGM.

The simulations described above considered the fluid in the single-screw mixer to be a Newtonian fluid. In a Newtonian fluid

E-mail address: James.Buick@port.ac.uk the viscosity, defined as the ratio of the stress to the velocity gradient of the fluid, is constant. In many practical situations the fluid in a single-screw extruder will exhibit non-Newtonian behaviour. NonNewtonian fluids have a viscosity which is not constant, it can vary with, for example, shear, temperature or time.

Here we will consider only shear dependent non-Newtonian fluids. A dilatant or shear-thickening fluid has an apparent viscosity which increases with increasing shear, for example corn starch, clay slurries and certain surfactants. A pseudoplastic or shear-thinning fluid has an apparent viscosity which decreases with increasing shear, for example polymer melts such a molten polystyrene, polymer solutions such as polyethylene oxide in water, paint and blood (Quarteroni et al., 2000).

A feature of the LBM is that it is suitable for simulating a nonNewtonian fluid. Gabbanelli et al. (2005) considered a power-law non-Newtonian fluid where the apparent viscosity was calculated as a function of the rate of strain which was found by differentiating the velocity field. The model was found to be first-order accurate for simple flows and was further applied to study flow in a reentrant corner geometry. Kehrwald (2005) considered an LBM for shearthinning fluids where the rate of strain was determined from known quantities without the need for differentiation. This model was applied to liquid composite moulding. Artoli and Sequeira (2006) also considered a model where the rate of strain was found without differentiating the velocity field. They applied their model to oscillating flows. Non-Newtonian simulations of blood flow using the LBM have also been considered by a number of authors (Ouared and Chopard, 2005; Artoli et al., 2006; Boyd and Buick, 2007; Boyd et al., 2007). 
It has been shown that second-order accuracy can be obtained using the LBM with a non-Newtonian viscosity described by a power-law model (Boyd et al., 2006). Preliminary results have shown qualitative differences between the velocity fields of a shear-thinning fluid and a Newtonian fluid in a single-screw extruder (Buick and Boyd, 2006).

The success of the LBM in simulating flow in a single-screw extruder and in simulating non-Newtonian fluids, coupled with the evidence that there is a significant difference between Newtonian and non-Newtonian flows in a screw-extruder, have motivated the present study. The LBM for a non-Newtonian fluid is described in Section 2. In Section 3 the validity of the model is investigated and simulation results are presented for a range of both shear-thinning and shear-thickening fluids.

\section{The lattice Boltzmann model}

The LBM (Chen and Doolen, 1998; Succi, 2001; Wolf-Gladrow, 2000) has recently been developed as an alternative technique for simulating fluid flow. Here we describe the Newtonian twodimensional D2Q9 model and the modifications required to simulate a power-law, non-Newtonian fluid.

\subsection{The D2Q9 LBM model}

The model evolves according to the kinetic equation

$f_{i}\left(\boldsymbol{x}+\boldsymbol{e}_{i}, t+1\right)-f_{i}(\boldsymbol{x}, t)=\Omega_{i}$

for $i=0,1, \ldots, 8$, where $f_{i}$ denotes the distribution function along direction $\boldsymbol{e}_{i}$ and

$\boldsymbol{e}_{0}=(0,0)$

$\boldsymbol{e}_{i}=\left(\cos \left(\frac{\pi}{2}(i-1)\right), \sin \left(\frac{\pi}{2}(i-1)\right)\right)$

for $i=1,2,3,4$ and

$\boldsymbol{e}_{i}=\sqrt{2}\left(\cos \left(\frac{\pi}{2}(i-1)+\frac{\pi}{4}\right), \sin \left(\frac{\pi}{2}(i-1)+\frac{\pi}{4}\right)\right)$

for $i=5,6,7,8$. The left-hand side of Eq. (1) represents streaming of the distribution functions at unit speed from one site $\boldsymbol{x}$ to a neighbouring site on a regular underlying grid defined by the link vectors $\boldsymbol{e}_{i}$. The right-hand side of Eq. (1) is the collision function which determines the manner in which the distribution functions interact at each site. The form of Eq. (1) makes the LBM discrete in both space and time.

The fluid density, $\rho$, and velocity, $\boldsymbol{u}$, are determined locally at each site and each time-step as follows:

$\rho(\boldsymbol{x}, t)=\sum_{i=0}^{i=8} f_{i}(\boldsymbol{x}, t) \quad$ and $\quad \rho(\boldsymbol{x}, t) \boldsymbol{u}(\boldsymbol{x}, t)=\sum_{i=0}^{i=8} f_{i}(\boldsymbol{x}, t) \boldsymbol{e}_{i}$.

Conservation of mass and momentum requires that the collision term, $\Omega_{i}$ in Eq. (1) satisfies

$\sum_{i=0}^{i=8} \Omega_{i}=0$ and $\sum_{i=0}^{i=8} \Omega_{i} \boldsymbol{e}_{i}=0$.

This is achieved in the LBM (Qian et al., 1992) using the Bhatnagar et al. (1954) equation

$\Omega_{i}=\frac{-1}{\tau}\left(f_{i}-\bar{f}_{i}\right)$,

which mimics the collisions by a relaxation towards an equilibrium distribution function $\bar{f}_{i}$ given by

$\bar{f}_{i}(\boldsymbol{r}, t)=\rho\left(1+3 \boldsymbol{e}_{i} \cdot \boldsymbol{u}+\frac{9}{2}\left(\boldsymbol{e}_{i} \cdot \boldsymbol{u}\right)^{2}-\frac{3}{2} u^{2}\right)$, where $w_{0}=\frac{4}{9}, w_{1}=w_{2}=w_{3}=w_{4}=\frac{1}{9}$ and $w_{5}=w_{6}=w_{7}=w_{8}=\frac{1}{36}$. The rate of relaxation is determined by the relaxation time $\tau$. Combining Eqs. (1) and (4) and performing a Taylor series expansion up to second order gives

$\left(\partial_{t}+\boldsymbol{e}_{i} \cdot \nabla\right) f_{i}+\frac{1}{2}\left(\partial_{t}+\boldsymbol{e}_{i} \cdot \nabla\right)^{2} f_{i}=\frac{-1}{\tau}\left(f_{i}-\bar{f}_{i}\right)$.

Introducing $\varepsilon$, the Knudsen number (Wolfram, 1986), which is the ratio of the mean free path to the characteristic length of the system; applying a Chapman-Enskog expansion (Frisch et al., 1987):

$f_{i}=\bar{f}_{i}+\varepsilon\left(f_{i}^{(1)}+\varepsilon f_{i}^{(2)}\right)$,

$\frac{\partial}{\partial t}=\frac{\partial}{\partial t_{1}}+\varepsilon \frac{\partial}{\partial t_{2}}$

$\frac{\partial}{\partial \boldsymbol{x}}=\frac{\partial}{\partial \boldsymbol{x}_{1}}$

and collecting terms up to second order in $\varepsilon$, leads to the mass and momentum equations (Chen and Doolen, 1998)

$\frac{\partial \rho}{\partial t}+\nabla \cdot \rho \boldsymbol{u}=0$

and

$\frac{\partial \rho u_{\alpha}}{\partial t}+\nabla_{\beta} \Pi_{\alpha \beta}=0$

where the momentum flux tensor is given by

$\Pi_{\alpha \beta}=\sum_{i}\left(\boldsymbol{e}_{i}\right)_{\alpha}\left(\boldsymbol{e}_{i}\right)_{\beta}\left[\bar{f}_{i}+\left(1-\frac{1}{2 \tau}\right) f_{i}^{(1)}\right]$.

Greek subscripts are used to represent vector components while Roman subscripts label the distribution functions, $f_{i}$ and $\bar{f}_{i}$, and link vectors, $\boldsymbol{e}_{i}$. Using the expression for the equilibrium distribution function, Eq. (5), gives

$\sum_{i}\left(\boldsymbol{e}_{i}\right)_{\alpha}\left(\boldsymbol{e}_{i}\right)_{\beta} \bar{f}_{i}=\rho u_{\alpha} u_{\beta}+\frac{1}{3} \rho \delta_{\alpha \beta}$

In a fluid with pressure $p$ and kinematic viscosity $v$ the momentum flux tensor takes the form

$\Pi_{\alpha \beta}=\rho u_{\alpha} u_{\beta}+p \delta_{\alpha \beta}-2 \rho v S_{\alpha \beta}$,

where $S_{\alpha \beta}$ is the strain tensor. Thus, expressing the pressure as $p=$ $c_{S}^{2} \rho$ we see that the speed of sound is $c_{s}=1 / \sqrt{3}$ and, following Artoli (2003),

$S_{\alpha \beta}=-\left(1-\frac{1}{\tau}\right) \frac{1}{2 \rho v} \sum_{i}\left(\boldsymbol{e}_{i}\right)_{\alpha}\left(\boldsymbol{e}_{i}\right)_{\beta} f_{i}^{(1)}$.

Evaluating Eq. (13) using the first-order Chapman-Enskog expansion of Eq. (6) gives (Chen and Doolen, 1998)

$\Pi_{\alpha \beta}=\rho u_{\alpha} u_{\beta}+p \delta_{\alpha \beta}-v\left(\frac{\partial \rho u_{\beta}}{\partial_{\alpha}}+\frac{\partial \rho u_{\alpha}}{\partial_{\beta}}\right)$,

where

$v=(2 \tau-1) / 6$.

In the incompressible limit, $\partial_{\alpha} \rho=0$, the fluid density can be removed from the derivatives in Eqs. (8) and (14). Thus the LBM scheme satisfies the continuity and Navier-Stokes equations for a Newtonian fluid with kinematic viscosity $v$. The value of the kinematic viscosity is determined by the free parameter $\tau$ (Eq. (15)) which is introduced in the collision function, Eq. (4). 


\subsection{Non-Newtonian simulations}

To simulate a shear dependent non-Newtonian fluid, it is necessary to determine the shear at each point in the simulation. This can be determined from the strain rate tensor

$S_{\alpha \beta}=\frac{1}{2}\left(\nabla_{\beta} u_{\alpha}+\nabla_{\alpha} u_{\beta}\right)$.

This would generally require the evaluation of the derivatives in Eq. (16). Using the LBM it is also possible to calculate $S_{\alpha \beta}$ from Eq. (13). That is, it can be found directly from the distribution functions without the need for significant extra computation.

Here we apply the power-law model for a non-Newtonian fluid where the apparent kinematic viscosity, $v_{a p}$ is determined as (Quarteroni et al., 2000; Quarteroni and Veneziani, 1997; Gijsen, 1998; Neofytou and Drikakis, 2003; Hussain et al., 1999)

$v_{\mathrm{ap}}(\dot{\gamma})=m|\dot{\gamma}|^{n-1}$,

where $m$ and $n$ are parameters that are determined by curve fitting equation (17) to physical viscometric data. $n<1$ corresponds to a shear-thinning fluid, $n>1$ represents a shear-thickening fluid and $n=1$ is the Newtonian limit. The shear rate, $\dot{\gamma}$ is determined form $S_{\alpha \beta}$ by

$\dot{\gamma}=2 \sqrt{D_{\mathrm{II}}}$,

where $D_{\mathrm{II}}$ is the second invariant of the strain rate tensor:

$D_{\mathrm{II}}=\sum_{\alpha, \beta=1}^{l} S_{\alpha \beta} S_{\alpha \beta}$,

where here $l=2$ for a two-dimensional simulation. The nonNewtonian fluid is then implemented in the LBM scheme by converting the local apparent viscosity $v_{\text {ap }}$ to a local apparent relaxation time $\tau_{\text {ap }}$ using Eq. (15). The LBM is implemented according to Eqs. (1) and (4) with $\tau$ replaced by $\tau_{\mathrm{ap}}(\boldsymbol{x}, t)$. by

A dimensionless number analogous to Reynold's number is given

$R e_{\mathrm{PL}}=\frac{U^{2-n} L^{n}}{m}$

where $U$ and $L$ are characteristic velocity and length scales, respectively.

The non-Newtonian LBM scheme was implemented using the following procedure:

1. Initialise the fluid domain by setting each of the distribution functions equal to their equilibrium value: $f_{i}(\boldsymbol{x}, t=0)=\bar{f}_{i}(\boldsymbol{x}, t=0)$. Here Eq. (5) is used with the initial density constant and the initial velocity zero.

2. Collision step

(a) Determine $\rho(\boldsymbol{x}, t)$ and $\boldsymbol{u}(\boldsymbol{x}, t)$ from Eq. (2).

(b) Determine $\tau(\boldsymbol{x}, t)$ from Eqs. (13), (19), (18), (17) and (15).

(c) Calculate the new distribution function using

$$
f_{i}^{*}(\boldsymbol{x}, t+1)=f_{i}(\boldsymbol{x}, t) \Omega_{i}
$$

in place of Eq. (1), combined with Eqs. (4) and (5).

3. Stream the new distribution functions to their neighbouring sites:

$$
f_{i}\left(\boldsymbol{x}+\boldsymbol{e}_{i}, t+1\right)=f_{i}^{*}(\boldsymbol{x}, t+1) .
$$

4. Proceed through the next time-step starting at step 2 .

\section{Results}

Within the LBM scheme there is a significant advantage in calculating the strain using Eq. (13) rather than using the traditional definition, Eq. (16). The advantage comes from the numerical efficiency of applying Eq. (13) compared to calculating the derivatives required in Eq. (16). When simulating a non-Newtonian fluid it is also important to consider the accuracy. This is shown in Fig. 1 for the case of Newtonian, two-dimensional, Poiseuille flow for which there is an analytic solution:

$u=\frac{G}{2 \mu}\left(a^{2}-y^{2}\right)$

where $G$ is the pressure gradient driving the flow and $\mu$ is the viscosity. The simulations of Poiseuille flow were run with a channel width $2 a$ of 20 lattice units. Fig. 1 shows the normalised strain (given by $S^{*}=(\mu / G a) S$ for Poiseuille flow) plotted against the normalised position $y^{*}=y / a$ where $S_{f}$ is calculated from Eqs. (13) and (19) as $S_{f}=\frac{1}{2} \sqrt{2 D_{\mathrm{II}}} ; S_{d}$ was obtained based on Eq. (16) as $S_{d}=\frac{1}{2} \partial u / \partial y$; and $S_{a}=-\frac{1}{2}(G / \mu) y$ is the analytic strain. Here $S$ represents $S_{x y}$. The derivatives required to determine $S_{d}$ were calculated from the LBM velocities using a central difference equation to determine the shear mid-way between the grid points. Also shown in Fig. 1 is the error function

$E_{S}=\left|\frac{S_{f}-S_{a}}{S_{a}}\right|$.

Fig. 1 shows good agreement between the different methods for calculating the strain. It also shows that, even for a relatively small channel, the error in calculating the strain using Eq. (13) is small.

Simulations of flow in a single-screw extruder were performed for shear-thinning, shear-thickening and Newtonian fluids. In a frame of reference moving with the rotating screw, the problem reduces to that of cavity flow with one moving wall and three stationary walls. The velocity can then be separated into a two-dimensional crosssection component and a stream-wise component. It is the crosssectional component, perpendicular to the spiral direction, which is considered here. This enables a direct comparison to be made with previous work and to enable comparison with theory for the Newtonian case. A computational grid of height $h$ and length $l$ was simulated with the top wall at $y=h$ moving with velocity $\left(u_{0}, 0\right)$ and

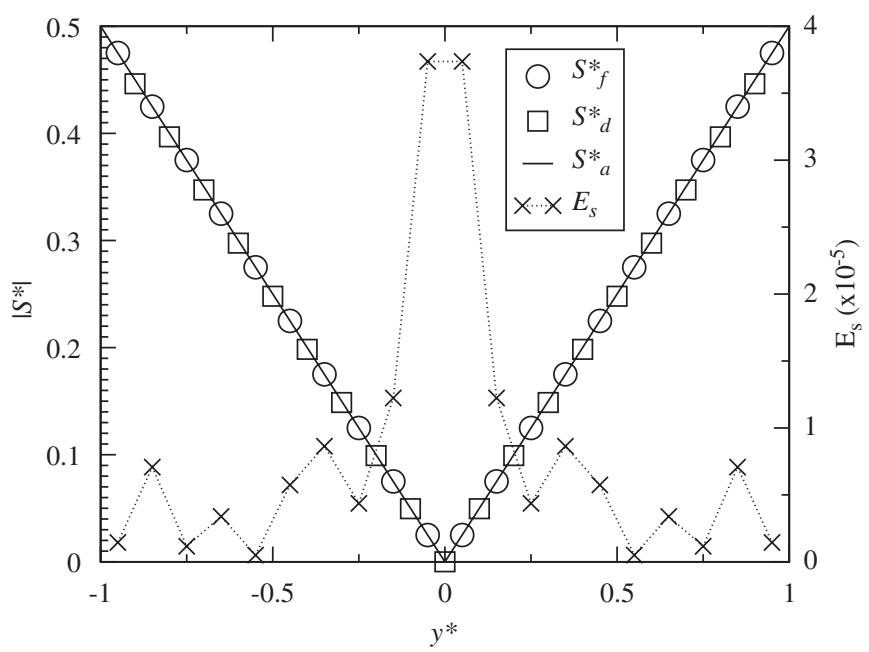

Fig. 1. The normalised strain for Newtonian Poiseuille flow calculated from the distribution functions $\left(S_{f}^{*}\right)$, a central difference equation $\left(S_{d}^{*}\right)$ and analytically $\left(S_{a}^{*}\right)$. Also shown is the relative error between $S_{f}^{*}$ and $S_{a}^{*}$. 

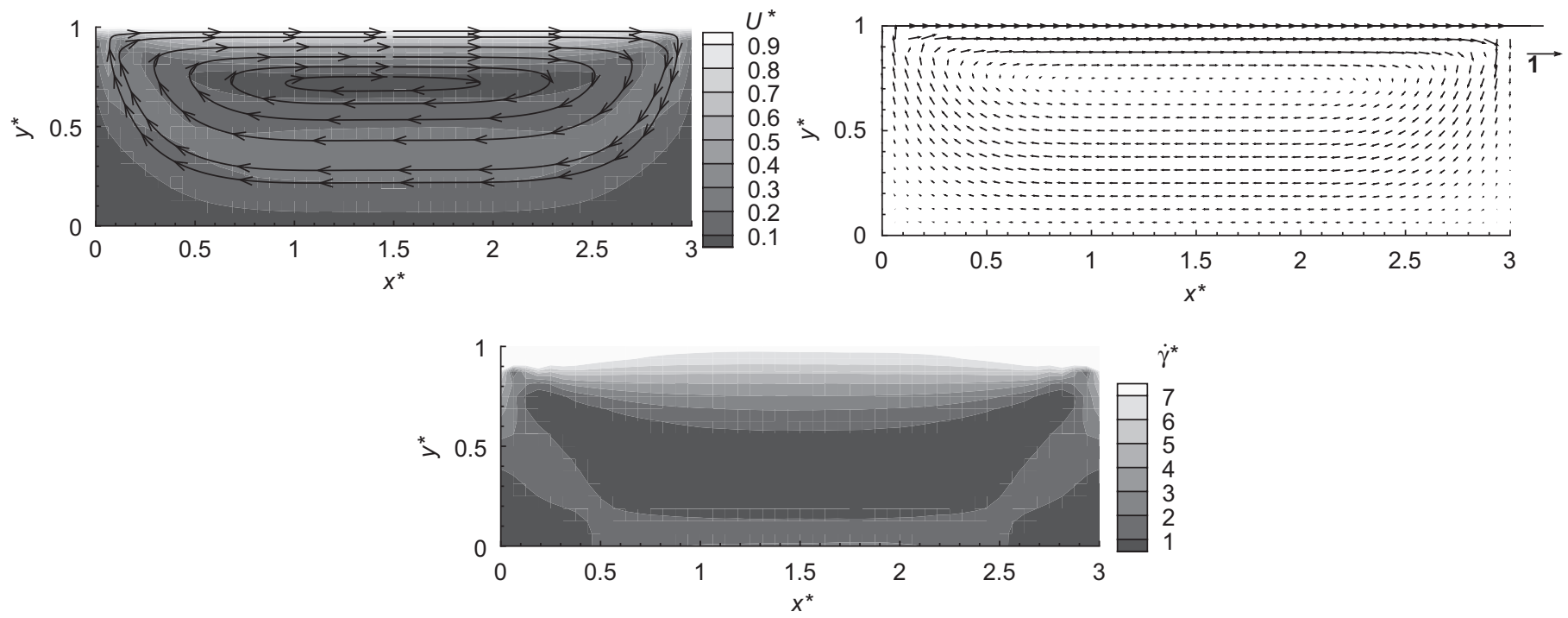

Fig. 2. Flow pattern in the mixing section of a screw extruder for $n=0.5$. (a) Velocity magnitude and streamlines, (b) velocity vector field, and (c) shear rate.

a

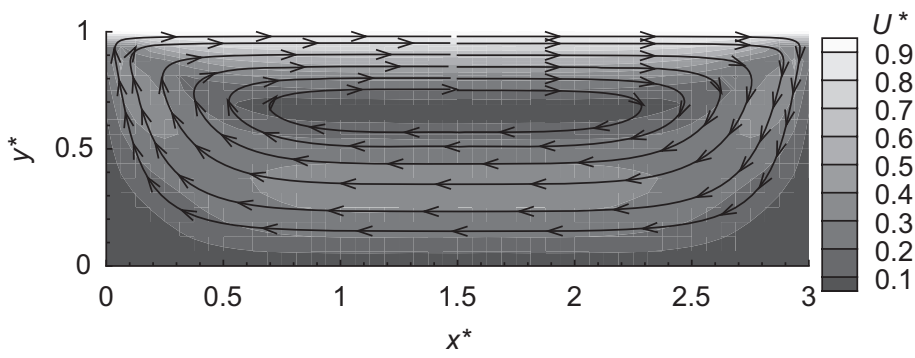

b

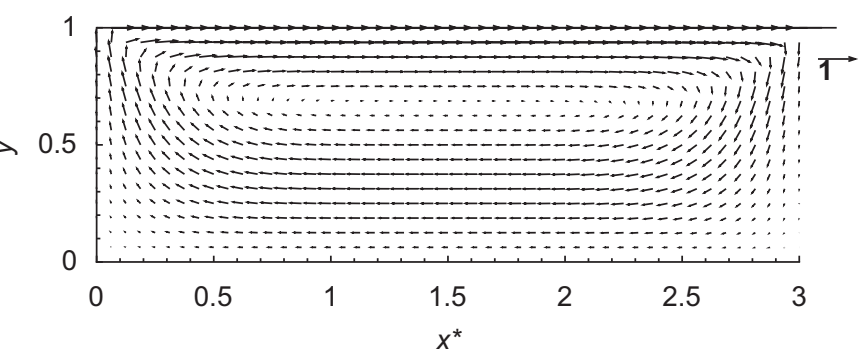

C

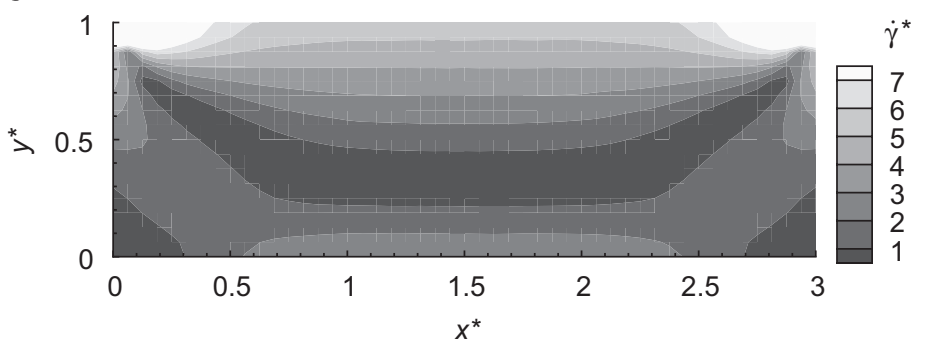

Fig. 3. Flow pattern in the mixing section of a screw extruder for $n=1.0$. (a) Velocity magnitude and streamlines, (b) velocity vector field, and (c) shear rate.

all the other walls stationary. The results are presented in terms of the normalised positions $x^{*}=x / h, y^{*}=y / h$ and velocities, $\left(u^{*}, v^{*}\right)=$ $\left(u / u_{0}, v / u_{0}\right)$. Here the ratio $x^{*} / y^{*}$ was fixed at 3 , the value used in earlier work. At the moving walls the boundary conditions were applied following Zou and He (1997). At stationary walls half-way bounce back boundary conditions were applied, see for example Zou and $\mathrm{He}$ (1997); these give a boundary half-way along the lattice link.

Figs. 2-4 show the results of simulations performed with $n=0.5$ (shear-thinning), $n=1.0$ (Newtonian) and $n=1.5$ (shear-thickening), respectively. The magnitude of the velocity, $U^{*}=\sqrt{\left(u^{*}\right)^{2}+\left(v^{*}\right)^{2}}$, and streamlines calculated from the flow are shown in part (a) of Figs. 2-4. The velocity and shear rate fields are depicted in parts (b) and (c), respectively. The results in Fig. 3, the Newtonian case, were found to be identical to results obtained using a true Newtonian model with a fixed viscosity. The results show that the velocity field varies significantly with the non-Newtonian nature of the fluid. This variation can be seen in more detail in Fig. 5 which shows the $x$ component of the velocity, $u^{*}$, as a function of the $y^{*}$-position along a cross-section through the centre of the screw extractor. Results are shown for $n=0.5,0.75,1.0,1.25$ and 1.5. Also shown are the results from a Newtonian LBM simulation and the analytic solution for a Newtonian fluid (McKelvey, 1962):

$u^{*}=y^{*}\left(2-3 y^{*}\right)$.

In each case the $x$-velocity decreases with distance from the bottom boundary to a minimum (negative) value. It then increases through zero to a normalised value of 1 at the top wall. Increasing $n$ (reducing the shear-thinning or increasing the shear-thickening behaviour) increases the magnitude of the velocity minimum which moves closer to the bottom boundary. The position of the zero velocity also moves 

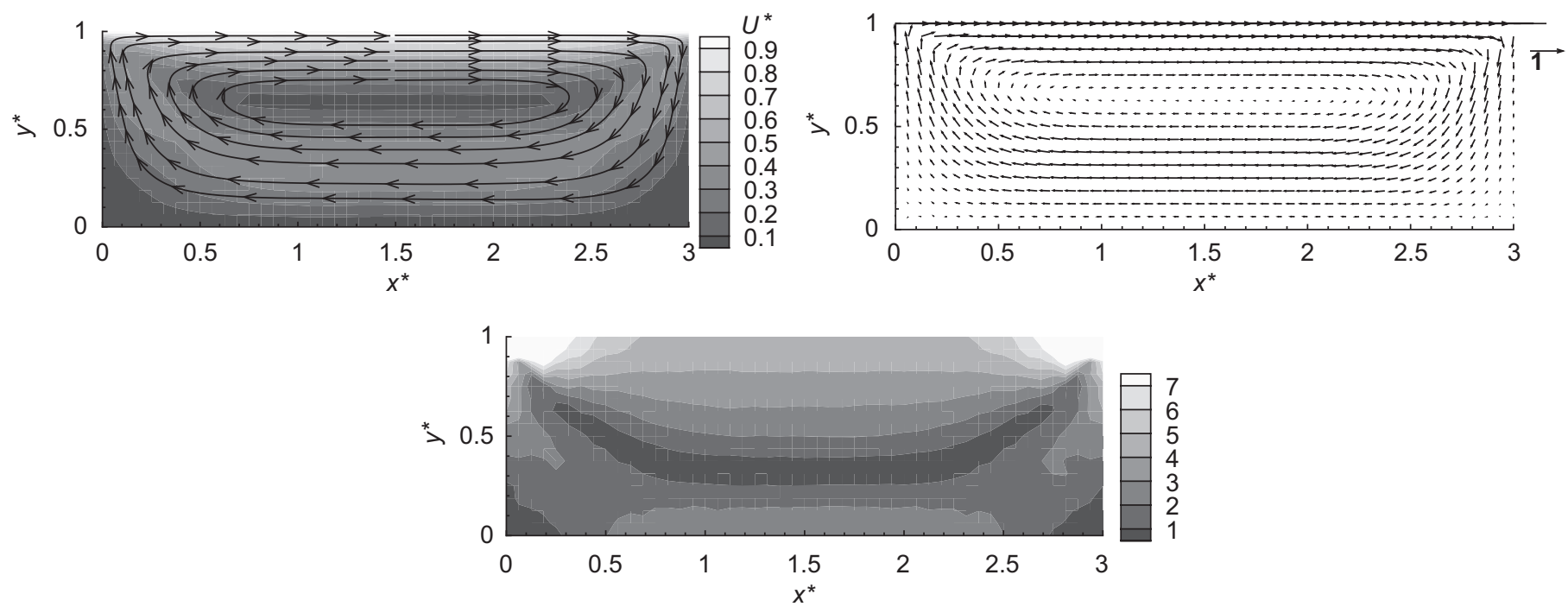

Fig. 4. Flow pattern in the mixing section of a screw extruder for $n=1.5$. (a) Velocity magnitude and streamlines, (b) velocity vector field, and (c) shear rate.

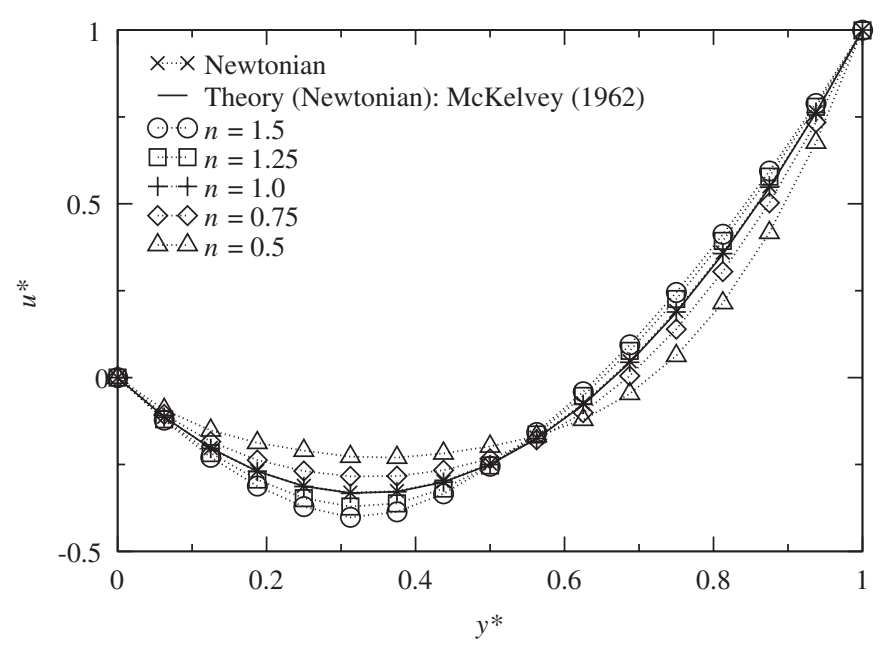

Fig. 5. The normalised $x$-component of the velocity as a function of the normalised $y$-position along a section through the centre of the computational domain.

closer to the bottom boundary and above this point the velocity increases. If the fluid is considered to consist of a top region where $u^{*}$ is positive and a bottom region where $u^{*}$ is negative, then increasing $n$ increases both the size of the top region and the velocity inside it. Consequently this reduces the size of the bottom region and increases the magnitude of the velocity minimum.

The variation in the normalised strain was also considered and is shown in Fig. 6. The results show the change in the strain with the non-Newtonian nature of the fluid. In the bottom $\frac{3}{4}$ of the extruder region the shear increases as a function of $n$ (shear-thinning to shearthickening). This trend is reversed in the top $\frac{1}{4}$. Fig. 6 also provides further validation of Eq. (13) for the strain rate tensor. The position of zero shear (corresponding to the velocity minimum) is also seen to move further from the bottom wall at low values of $n$. This is consistent with the velocity observations above.

\section{Discussion}

Simulations have been presented for both shear-thinning and shear-thickening non-Newtonian fluids described by a power-law,

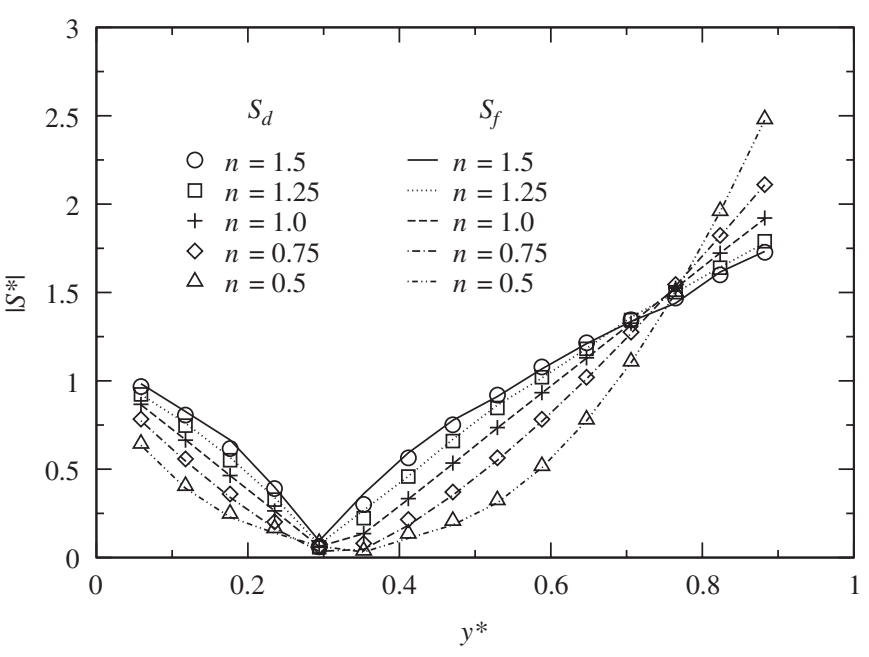

Fig. 6. The normalised shear as a function of the normalised $y$-position along a section through the centre of the computational domain. The shear $S_{d}$ is calculated using Eq. (16) and $S_{f}$ is calculated using Eq. (13).

Eq. (17). The power-law is commonly used because of its simplicity and because it is often a good approximation to the behaviour of a real fluid. For some fluids, such as a Bingham fluid, the form of the power-law will not be suitable and an alternative expression is required. In particular, the form of Eq. (17) suggests that for $n<1$, the apparent viscosity will be infinite at rest and will approach zero as the shear approaches infinity. In practice a non-Newtonian fluid will have a maximum and minimum apparent viscosity. Despite this, the power-law can still provide a good approximation over the range of shears present in a simulation. If the power-law is not suitable for a particular non-Newtonian fluid, then there are a number of alternative laws available which may provide a better approximation. It is also known that some non-Newtonian fluids have a non-zero yield stress, $\tau_{0}$. Such fluids can be simulated using the simplified Herschel and Bulkley (1926) equation where the apparent viscosity is given by a power-law with the addition of a yield stress term:

$v_{\mathrm{ap}}(\dot{\gamma})=\frac{1}{\rho} \tau_{0} \dot{\gamma}^{-1}+m \dot{\gamma}^{n-1}$ 
The Herschel-Bulkley model, Eq. (26), has been shown to be particularly suitable for polymer solutions and suspensions (Hong et al., 2007), while molten polymer is better described (Hong et al., 2006) by the Carreau et al. (1979) model:

$$
\frac{v_{\mathrm{ap}}(\dot{\gamma})-v_{\infty}}{v_{0}-v_{\infty}}=\left[1+(\lambda \dot{\gamma})^{2}\right]^{(n-1) / 2}
$$

where $\lambda$ is a constant, $v_{0}$ is the zero-shear (Newtonian) viscosity and $v_{\infty}$ is a limiting viscosity at high shear rates. For polymer melt $v_{\infty}$ is commonly taken to be zero in which case Eq. (27) approaches a Newtonian fluid at low shear rate and a power-law fluid at high shear rate. There is no limitation on the form of the non-Newtonian law which can be applied in the LBM approach. The desired law can be substituted for Eq. (17) (Boyd et al., 2007).

The form of the velocity field depicted in Fig. 3 for the Newtonian case is consistent with previous studies (Yao et al., 1996, 1997; Horiguchi et al., 2003) using the same parameters. Experimental measurements are often presented in the curved geometry of the extruder, see for example Agemura et al. (1995), making a direct quantitative comparison difficult. Non-Newtonian fluids have received less attention. Gabriele et al. (2001) considered a liquorice extract which was shown to be a shear-thinning fluid described well by the power-law, Eq. (17), with $n \simeq 0.37$. A number of results were presented for different pressure gradients which all showed the same trend. Cross channel profiles showed a negative velocity for $y^{*}$ less than approximately 0.8 with a minimum velocity of $u^{*} \simeq-0.15$. Despite the different geometry it is clear that these values are different to the Newtonian values and show the same trend as the results presented here. That is, for decreasing values of $n$, larger regions of negative velocity were observed with a correspondingly smaller absolute value for the velocity minimum in this region. Numerical simulation using the finite-volume method have been considered by Khalifeh and Clermont (2005) for a number of different fluids including a Newtonian and a shear dependent non-Newtonian fluid similar to the one presented here. The Carreau law, Eq. (27) was used to describe the shear-thinning fluid where $v_{\infty} / v_{0}=0.1, \lambda=0.55$ and $n$, which plays a similar role to the parameter in the powerlaw model but cannot be directly compared, takes the value 0.44 . Khalifeh and Clermont (2005) were primarily interested in modelling thermal gradients but also present limited results for the isothermal case. The Newtonian and non-Newtonian models are qualitatively similar to the results presented here; however, a detailed comparison such as presented in Figs. 5 and 6 is not possible. A finite element simulation of memory-integral type fluids was performed by Broszeit (1997) for a single-screw extruder. Again qualitative similarities were evident but the differences in the simulation and the type of fluid prevent a detailed qualitative comparison.

Figs. 2-5 clearly demonstrate a change in the velocity field inside the mixing section of the screw extruder with the nonNewtonian nature of the simulated fluid. Further work is required to determine how this change in the flow will influence the mixing properties of the extruder. This could be determined following, for example, Camesasca et al. (2006). It is also evident from Fig. 6 that the shear profile inside the mixing section is also dependent on the non-Newtonian properties of the fluid. Here the simulation of a shear-thinning fluid with $n=0.5$ was observed to have a maximum shear in excess of $25 \%$ greater than a Newtonian fluid. This has implications when a high shear rate can damage the material being mixed.

\section{Conclusion}

Numerical simulation of the flow field in the mixing section of a single-screw extruder has been considered for a range of nonNewtonian fluids. This was done using the lattice Boltzmann model which simulates the fluid using a simplified kinetic equation. A power-law model was used to simulate non-Newtonian fluids with a shear dependent apparent viscosity. In the LBM this was implemented by calculating the shear in a local manner directly from the distribution functions which describe the fluid. The accuracy of this approach was tested for Newtonian Poiseuille flow by comparing the simulations to the known analytic solution as well as considering the traditional approach of calculating the shear from velocity gradients. Excellent agreement was found in both cases.

Simulations of the velocity and shear rate in the mixing section were then presented for a shear-thinning and a shear-thickening fluid as well as a Newtonian fluid for comparison. A more detailed analysis of the different flow parameters was also performed for a wider range of non-Newtonian fluids on a section through the centre of the screw-extruder.

The results identified the manner in which the non-Newtonian nature of a fluid changes the flow pattern in the simple geometry considered; and highlights the need to fully consider the nonNewtonian nature of the fluid in such a simulation. The LBM has been shown to be effective for simulating such problems in terms of both the general approach and the local calculation of the shear from the distribution functions. This method for calculating the shear provides a significant advantage in terms of computational efficiency when compared with calculating the shear from velocity derivatives-an approach which must be taken in alternative numerical schemes. This computational efficiency would be further enhanced if a parallel implementation was considered for a larger simulation.

The dependence of the velocity field on the non-Newtonian nature of the fluid has important implications for fluid mixing. The need to consider non-Newtonian fluids when simulating fluid mixing has been highlighted and a numerical scheme for doing this has been presented and investigated.

\section{References}

Agemura, C.K., Kauten, R.J., McCarthy, K.L., 1995. Flow fields in straight and tapered screw extruders using magnetic resonance imaging. Journal of Food Engineering $25,55-72$.

Artoli, A., 2003. Mesoscopic computational haemodynamics. Ph.D. Thesis, University of Amsterdam.

Artoli, A.M., Sequeira, A., 2006. Mesoscopic simulations of unsteady shear-thinning flows. Springer Lecture Notes in Computer Science, vol. 3992. Springer, Berlin, pp. $78-85$.

Artoli, A.M., Sequeira, A., Janela, J., 2006. Shear-thinning viscosity effects in bifurcating blood vessels. Journal of Biomechanics 39, S310.

Bhatnagar, P.L., Gross, E.P., Krook, M., 1954. A model for collision processes in gases. I. Small amplitude processes in charged and neutral one-component systems. Physical Review 94, 511-525.

Boyd, J., Buick, J.M., 2007. Comparison of Newtonian and non-Newtonian flows in a two-dimensional carotid artery model using the lattice Boltzmann method. Physics in Medicine and Biology 52, 6215-6228.

Boyd, J., Buick, J., Green, S., 2006. A second-order accurate lattice Boltzmann nonNewtonian flow model. Journal of Physics A 39, 14241-14247.

Boyd, J., Buick, J.M., Green, S., 2007. Analysis of the Casson and Carreau-Yasuda non-Newtonian blood models in steady and oscillatory flows using the lattice Boltzmann method. Physics of Fluids 19, 093103.

Broszeit, J., 1997. Finite-element simulation of circulating steady flow for fluids of the memory-integral type: flow in a single-screw extruder. Journal of NonNewtonian Fluid Mechanics 70, 35-58.

Buick, J.M., Boyd, J., 2006. Non-Newtonian shear thinning flow in a single-screw extruder. WSEAS Transactions on Fluid Mechanics 1, 844-859.

Buick, J.M., Cosgrove, J.A., 2006. Numerical simulation of the flow field in the mixing section of a screw extruder by the lattice Boltzmann model. Chemical Engineering Science 61, 3323-3326.

Camesasca, M., Kaufman, M., Manas-Zloczower, I., 2006. Quantifying fluid mixing with the Shannon entropy. Macromolecular Theory and Simulations 15, 595-607.

Carreau, P.J., De Kee, D., Daroux, M., 1979. An analysis of the viscous behaviour of polymeric solutions. Canadian Journal of Chemical Engineering 57, 135-141.

Chen, S., Doolen, G.D., 1998. Lattice Boltzmann method for fluid flows. Annual Review of Fluid Mechanics 30, 329-364.

Frisch, U., d'Humières, D., Hasslacher, B., Lallemand, P., Pomeau, Y., Rivet, J.-P., 1987. Lattice gas hydrodynamics in two and three dimensions. Complex Systems 1, 649-707.

Gabbanelli, S., Drazer, G., Koplik, J., 2005. Lattice Boltzmann method for nonNewtonian (power-law) fluids. Physical Review E 72, 046312. 
Gabriele, D., Curcio, S., de Cindio, B., 2001. Optimal design of single-screw extruder for liquorice candy production: a rheology based approach. Journal of Food Engineering 48, 33-44.

Gijsen, F.J.H., 1998. Modeling of wall shear stress in large arteries. Ph.D. Thesis, Eindhoven University of Technology.

Herschel, W.H., Bulkley, R., 1926. Konsistenzmessungen von Gummi-Benzollösungen. Kolloid-Zetischr 39, 291.

Hong, R., Chen, L.L., Li, H.Z., 2006. On contraction flow of polystyrene melt through slit dies. Computers and Applied Chemistry 23, 1069-1072 (in Chinese with English abstract).

Hong, R.Y., Ren, Z.Q., Han, Y.P., Li, H.Z., Zheng, Y., Ding, J., 2007. Rheological properties of water-based $\mathrm{Fe}_{3} \mathrm{O}_{4}$ ferrofluids. Chemical Engineering Science 62, 5912-5924.

Horiguchi, H., Takahashi, K., Tokota, T., 2003. Numerical simulation of the flow field in the mixing section of a screw extruder by the lattice gas automata method. Journal of Chemical Engineering Japan 36, 110-113.

Hussain, M.A., Kar, S., Puniyani, R.R., 1999. Relationship between power law coefficients and major blood constituents affecting the whole blood viscosity. Journal of Biosciences 24, 329-337.

Kehrwald, D., 2005. Lattice Boltzmann simulation of shear-thinning fluids. Journal of Statistical Physics 121, 223-237.

Khalifeh, A., Clermont, J.-R., 2005. Numerical simulations of non-isothermal threedimensional flows in an extruder by a finite-volume method. Journal of NonNewtonian Fluid Mechanics 126, 7-22.

McKelvey, J.M., 1962. Polymer a Processing. Wiley, New York, USA. pp. 228-247.

Neofytou, P., Drikakis, D., 2003. Non-Newtonian flow instability in a channel with a sudden expansion. Journal of Non-Newtonian Fluid Mechanics 111, 127-150.
Ouared, R., Chopard, B., 2005. Lattice Boltzmann simulations of blood flow: nonNewtonian rheology and clotting processes. Journal of Statistical Physics 121 209-221.

Qian, Y.H., D'Humières, D., Lallemand, P., 1992. Lattice BGK models for Navier-Stokes equation. Europhysics Letters 17, 479-484.

Quarteroni, A., Veneziani, A., 1997. Computational science for the 21st century. In: Bristeau, M.-O., Etgen, G., Fitzgibbon, W., Lions, J.L., Periaux, J., Wheeler, M.F. (Eds.), Modeling and Simulation of Blood Flow Problems. Wiley, New York.

Quarteroni, A., Tuveri, M., Veneziani, A., 2000. Computational vascular fluid dynamics: problems, models and methods. Computing and Visualisation in Science 2, 163-197.

Succi, S., 2001. The Lattice Boltzmann Equation-For Fluid Dynamics and Beyond Oxford University Press, Oxford.

Wolf-Gladrow, D.A., 2000. Lattice-gas cellular automata and lattice Boltzmann models. In: Dold, A., Takens, F., Teissier, B. (Eds.), Lecture Notes in Mathematics, vol. 1725. Springer, Berlin.

Wolfram, S., 1986. Cellular automaton fluids 1: basic theory. Journal of Statistical Physics 45, 471-529.

Yao, W.G., Takahashi, K., Abe, Y., 1996. Analytical study on flow and distributive mixing of a new type pin mixing section for screw extruder. International Polymer Proceedings XI, 222-227.

Yao, W.G., Takahashi, K., Koyama, K., Dai, G.C., 1997. Design of a new type pin mixing section for a screw extruder based on analysis of flow and distributive mixing performance. Chemical Engineering Science 52, 13-21.

Zou, Q., He, X., 1997. On pressure and velocity boundary conditions for the lattice Boltzmann BGK model. Physical Review E 9, 1591-1598. 